\title{
Novel fructooligosaccharides of Dioscorea alata L. tuber have prebiotic potentialities
}

Biplab Bandyopadhyay

Siksha Bhavana, Visva-Bharati

Prashanta Kumar Mitra

University of Gour Banga

Vivekananda Mandal ( $\nabla$ mandalvivek@gmail.com )

University of Gour Banga https://orcid.org/0000-0001-6523-8069

Narayan Chandra Mandal ( $\nabla$ mandalnc@rediffmail.com )

Visva-Bharati https://orcid.org/0000-0003-1631-0794

\section{Research Article}

Keywords: Dioscorea alata L., Fructooligosaccharides, Hypocholesterolemic, Probiotic LAB, Prebiotic index, Synbiotics

Posted Date: July 23rd, 2021

DOl: https://doi.org/10.21203/rs.3.rs-735703/v1

License: (c) (1) This work is licensed under a Creative Commons Attribution 4.0 International License. Read Full License 


\section{Novel fructooligosaccharides of Dioscorea alata L. tuber have prebiotic potentialities}

Authors: Biplab Bandyopadhyay ${ }^{1,2}$, Prashanta Kumar Mitra ${ }^{2}$, Vivekananda Mandal ${ }^{* 2}$, and Narayan Chandra Mandal $^{* 1}$

Mycology and Plant Pathology Laboratory, Department of Botany, Siksha Bhavan, Visva-Bharati, Santiniketan 71235, W.B., India

$\&$

Plant and Microbial Physiology and Biochemistry Laboratory, Department of Botany, University of Gour Banga, Malda - 732103, W. B., India

\section{Authors and their affiliations:}

Biplab Bandyopadhyay' Research Scholar, Mycology and Plant Pathology Laboratory, Department of Botany, Siksha Bhavan, Visva-Bharati, Santiniketan - 71235, W.B., India; and Plant and Microbial Physiology and Biochemistry Laboratory, Department of Botany, University of Gour Banga, Malda - 732103, W. B., India; Email: banerjeerev@gmail.com

Prashanta Kumar Mitra, Research Scholar, Plant and Microbial Physiology and Biochemistry Laboratory, Department of Botany, University of Gour Banga, Malda - 732103, W. B., India; pkmitrabot@gmail.com

Vivekananda Mandal, Profesor of Botany, Plant and Microbial Physiology and Biochemistry Laboratory, Department of Botany, University of Gour Banga, Malda - 732103, W. B., India; Email: mandalvivek@gmail.com

Narayan Chandra Mandal, Professor of Botany, Mycology and Plant Pathology Laboratory, Department of Botany, Siksha Bhavan, Visva-Bharati, Santiniketan-71235, W.B., India; Email: mandalnc@rediffmail.com

${ }^{* 1}$ Corresponding author: Narayan Chandra Mandal, Mycology and Plant Pathology Laboratory, Department of Botany, Siksha Bhavan, Visva-Bharati, Santiniketan - 71235, W.B., India; Phone: +91 9434016026; Email: mandalnc@rediffmail.com; ORCID: https://orcid.org/0000-0003-1631-0794

\footnotetext{
${ }^{* 2}$ Co-corresponding author: Vivekananda Mandal, Plant and Microbial Physiology and Biochemistry Laboratory,
} Department of Botany, University of Gour Banga, Malda - 732103, W. B., India; Phone no.: +91 9434556340 
(Home); 03512223664 (Office); Fax: (+91 3512) 223 568; Email: mandalvivek@gmail.com \& vivek.bot@ugb.ac.in; ORCID: https://orcid.org/0000-0001-6523-8069

\begin{abstract}
Dioscorea alata L. (Dioscoreaceae) is a versatile and popular tuber crop vegetable used across the globe. The main aim of the study is to extract and characterize the fructooligosaccharides (FOSs) fractions from the tuber of this plant and evaluate their prebiotic properties. The soluble FOSs were fractionated by the conventional solid-phase extraction method and were characterized by FT-IR, HPTLC, and ESI-MS analyses. In addition, the beneficial health attributes of these fractions were checked for prebiotic properties in vitro and in vivo in Swiss albino mice. The FT-IR, HPTLC, and ESI-MS analyses of the FOSs from the hot water and $80 \%$ ethanol fractions showed that both are rich in inulinlike prebiotics. Furthermore, these FOSs showed an excellent prebiotic activity score comparable to standard prebiotics, inulin. In addition, these FOSs had enhanced in vitro cholesterol-binding activities in the presence of probiotics LAB strains and had antioxidant efficacies. In vivo treatment in Swiss albino mice, these FOSs had significantly lowered total serum cholesterol, serum LDL, serum VLDL, triglyceride, and significantly enhanced serum HDL level and enhanced IgA mediated immunomodulation. The results were also more significant than the standard prebiotics, inulin. They also influenced the gut colonization of probiotic LAB strains. Thus, the study revealed that the FOSs from the storage tuber of $D$. alata possess some novel prebiotic that could contribute to human nutrition. This is the first report of the prebiotic FOS characterization from this plant.
\end{abstract}

Keywords: Dioscorea alata L.; Fructooligosaccharides; Hypocholesterolemic; Probiotic LAB; Prebiotic index; Synbiotics

\title{
1. Introduction
}

Human beings are called "meta-organisms" due to co-evolution with the vast number of micro-symbionts living in our bodies. The majority of these bacterial symbionts inhabit the gastrointestinal tract and constitute the human intestinal microbiota. The inhabited microbial communities provide metabolic, immunologic, and protective functions that play a crucial role in human health [1]. These beneficial microbial communities also influence the host physiology and living conditions, are popularly termed probiotics (WHO/FAO, 2006). Furthermore, the non-digestible dietary 
fibres with a low degree of polymerization (DP) selectively impose a positive impact on the composition, livingness, and metabolic activities of bacterial communities present in the human gut, commonly termed prebiotics $[2,3]$. There are many types of prebiotics. The majority of them are a subset of carbohydrate groups and are primarily oligosaccharides [4]. Among these, fructooligosaccharides (FOSs) are composed of linear chains of fructose units linked by $\beta 2-1$ bonds. The number of fructose units ranges from 2 to 60 and often terminate in a glucose unit [5]. FOSs are of low-calorific oligosaccharides used as alternative sweeteners in beverages, syrups, and foods. FOSs have GRAS certification (https://www.fda.gov/media/116858/download) and are often used in baby formula to help feed the beneficial bacteria that the intestines need. FOSs are a group of soluble fibres, and it helps absorb water in the intestines and give the faecal matter a consistent shape. Thus, an increasing level of soluble FOS fibre in the diet has been shown to reduce or eliminate digestive issues, such as constipation or diarrhoea. The introduction of FOS into a calorie-restricted diet also helped to reduce weight gain. For people with prediabetes and type 2 diabetes, FOS has the potential ability to balance a person's blood sugar $[6,7]$. Moreover, FOS usage also stimulated insulin secretion in the pancreas $[8,9]$. FOS is not carcinogenic, and it has little potential to disrupt or damage normal cell growth. Thus, consuming FOS will not feed cancer cells or contribute to their growth; instead, short-chain fructo-oligosaccharides (sc-FOS) protect colon carcinogenesis in animal models $[5,10,11]$. Thus, the diet rich in FOS could be a critical factor to modulate the gastrointestinal microbiota's composition and metabolism[11-14].

Different ethnic groups have traditional uses of many unique food items in their daily diet. However, many of these food commodities' detailed composition and beneficial health attributes have not been discovered. The storage tubers are such cheap and nutritious food sources that humankind has used since time immemorial. In this connection, yam's tubers (Dioscorea spp.), which the different communities across the globe have used, have brought attention to explore its health-beneficial attributes. The plant Dioscorea, belonging to the family Dioscoreaceae, is a vast genus covering 1559 nos. of plant species[15]. Many studies have explored that D. alata L., commonly known as Water Yam, has nutritional superiority compared with other tropical root crops [16]. It is mainly composed of starch (75-84\% of the dry weight) with a small number of proteins, lipids and vitamins and is very rich in minerals. Rural people have used different Dioscorea species due to their nutritional and antioxidant content [16-18]. The tuber powder is used to cure piles, reduce weakness, or kill stomach worms. Alves et al. [19] studied the gelling properties of extruded yam (D. alata) starch and concluded that the extruded starch might be applied in instant creams and puddings. Chou et al. [20] 
has explored the effects of storage temperatures on the composition and antioxidative activities of one kind of Taiwanese yam tubers (D. alata) and observed no significant change in DPPH radical-scavenging activity up to 11 weeks storage at room temperature. This indicates that this yam flour's health-beneficial attributes can be stored as

ready staple crop flour for such an extended time. In a study, Hsu et al. [21] established that the crude ethanolic extracts from $D$. alata peel showed more protection on tert-butyl hydroperoxide-induced oxidative stress in mouse liver cells, Hepa 1-6 and FL83B liver cells. Therefore, this evidence shows that the storage products of Yam tubers have many health beneficial attributes. Moreover, in a recent study, Liu et al. [22] reported that the yam flour might substitute wheat flour in wheat bread preparation, and the preparation has decreased the digestibility of wheat flour starch. They speculated that this low digestibility of the yam flour might be due to more thermal processing-resistant, intact starch granules.

In this connection, the present research reports on the characterization of the extractable soluble dietary FOSs fractions from D. alata L. and assess their effects on the lipid profile and microbial colonization in the gut of Swiss albino mice, either alone or in combination with probiotic LAB strains.

\section{Materials and methods}

\subsection{Plant sample}

The tuber of Dioscorea alata L. (Dioscoreaceae) was collected from the local market of Malda town, West Bengal, India. The voucher specimen (Ref. No. CNH/Tech.II/2018/77; dated 05-10-2018) was authenticated by the Botanical Survey of India, CNH, Shibpur, Howrah, India. The fresh samples were washed under tap water and then peeled to remove the inedible outer layer, chopped into smaller slices, and oven-dried at $60{ }^{\circ} \mathrm{C}$ for $4-5$ days. The dried samples were ground to powder by the mechanical grinder and were kept at room temperature $\left(\sim 30^{\circ} \mathrm{C}\right)$ until use.

\subsection{Extraction of carbohydrate fractions and assessment of in vitro prebiotic efficacy}

The oligosaccharide fraction was harvested from $20 \mathrm{~g}$ of powder sample using $200 \mathrm{ml}$ of different extractive solvents, viz. hot-water, cold-water, hot-acid $\left\{0.2(\mathrm{~N}), 0.5(\mathrm{~N}), 0.8 \mathrm{~N} \mathrm{H}_{2} \mathrm{SO}_{4,}\right\}$, hot-alkali $(1 \% \mathrm{NaOH})$, and hot and cold $80 \%$ ethanol for $1 \mathrm{hr}$ following the protocol of Bandyopadhyay et al. [23]. The fractions were dried in a rotary vacuum evaporator (R-25, Superfit, Mumbai, India) and coded as Cold-Wt, Hot-Wt, $0.2(\mathrm{~N}) \mathrm{H}_{2} \mathrm{SO}_{4}, 0.5(\mathrm{~N}) \mathrm{H}_{2} \mathrm{SO}_{4}, 0.8(\mathrm{~N})$ $\mathrm{H}_{2} \mathrm{SO}_{4}, 1 \% \mathrm{NaOH}$, and $80 \%$ Et-OH. 
The in vitro prebiotic efficacy (Prebiotic activity, cholesterol removal potentiality, and antioxidant efficiency) of the extracted carbohydrate fractions was done following Bandyopadhyay et al. [23]. Two probiotic LAB strains viz., Lactococcus lactis LAB-17 (GenBank Ac. No.: MK215681) and L. lactis LAB B 1.1 (GenBank Ac. No.: MK215683) were used for the synbiotic study. In addition, the enteric pathogenic strain E. coli MTCC 1667, purchased from the Microbial Type Culture Collection and Gene Bank, Chandigarh, India, was used as a non-probiotic challenge strain in this study. Here, inulin (standard prebiotics) (Hi-Media, India) was used as the positive control.

\subsection{Chemical characterization of the selected oligosaccharide fractions}

The FT-IR analyses (Perkin Elmer Spectrum 100 Series, USA), monosaccharide composition (HPTLC Silica gel 60 $\mathrm{F}_{254}$ sheets, Merck Darmstadt, Germany using CAMAG HPTLC system), and Electrospray Ionization Mass Spectrometry (ESI-MS, Advion Compact Mass spectrometer, Germany) in a positive ionization mode of the carbohydrate fractions were done following Bandyopadhyay et al. [23].

\subsection{In vivo health attributes of the carbohydrate fractions: Prebiotics and synbiotics}

Male Swiss albino mice with an average body weight between 40-60 g were taken for in vivo experimentation and were maintained following the Institutional Animal Ethics Committee guideline, Visva-Bharati University, India (vide Ref. No.: IACE/VB/2018/6; Dated: 24-04-2018). The mice were fed with the carbohydrate fractions at a dose of 40 $\mathrm{mg} / \mathrm{Kg}$ body weight/day along with a regular diet for 30 days. In addition, the live probiotic strains were also fed at a dose of $10^{8} \mathrm{CFU} / \mathrm{ml}$ per day.

The serum lipid profile (total serum cholesterol, total triglycerides, HDL, LDL, and VLDL levels, by Avantor RFCL Kit, Ref-83LS100-60); serum blood sugar level (GOD-POD method, by Siemens Analyser Kit, Ref- 28558); liver toxicity (viz. Serum glutamic pyruvic transaminase (SGPT) and serum glutamic oxaloacetic transaminase (SGOT), by Autospan Kit, Ref-PTC025); and renal toxicity (serum creatinine), by Avantor RFCL Kit, Ref-15000777) and serum Immunoglobulin A (by Abcam's IgA ELISA Kit, Ref- ab196263) of the experimental animal model were measured following Bandyopadhyay et al. [23].

\subsection{Influence on the gut colonization of probiotic LAB strains}

A scanning electron microscopic (SEM, Zeiss EVO 18, Germany) study of the small intestine (jejunum) of the experimental animals was performed to examine gut colonization of the probiotic LAB strains after selective feeding 
of carbohydrate fractions as mentioned above following Bandyopadhyay et al. [23]. The values were compared with the untreated (a negative control) and inulin treated (positive control) mice sets.

\subsection{Statistical analysis}

All the experiments were repeated thrice, and the average of the arithmetic mean \pm SE was calculated. Microsoft Excel and the Past (4.0.1) software were used to represent the data's graphical section.

\section{Results and discussion}

\subsection{Extractable fructooligosaccharides and prebiotic activity score}

Among the seven different crude carbohydrate fractions, the highest total carbohydrate content $(282.3 \mathrm{mg} / \mathrm{g}) \mathrm{was}$ found in $0.5(\mathrm{~N}) \mathrm{H}_{2} \mathrm{SO}_{4}$ fraction followed by $0.8(\mathrm{~N}) \mathrm{H}_{2} \mathrm{SO}_{4},(240.01 \mathrm{mg} / \mathrm{g})$ \& $0.2(\mathrm{~N}) \mathrm{H}_{2} \mathrm{SO}_{4}(178.03 \mathrm{mg} / \mathrm{g})$ fractions, respectively. However, hot water fraction $(112.08 \mathrm{mg} / \mathrm{g}), 80 \%$ ethanol fraction $(68.12 \mathrm{mg} / \mathrm{g})$, and hot $1 \% \mathrm{NaOH}$ fraction $(53.04 \mathrm{mg} / \mathrm{g})$ showed a considerable less amount of carbohydrate, and the lowest content was recovered in cold water fraction as shown in Fig. S1. The influence of the carbohydrate fractions on the growth of both the probiotics strains showed that the $0.8(\mathrm{~N}) \mathrm{H}_{2} \mathrm{SO}_{4}, 0.2(\mathrm{~N}) \mathrm{H}_{2} \mathrm{SO}_{4}$, and hot $1 \% \mathrm{NaOH}$ fractions had more activity than hot water and $80 \%$ ethanol fractions (Fig. S2). A higher prebiotic index was observed in hot water and $80 \%$ ethanol fractions comparable to the standard prebiotic inulin (Fig. 1). Though the $0.8(\mathrm{~N}) \mathrm{H}_{2} \mathrm{SO}_{4}, 0.2(\mathrm{~N}) \mathrm{H}_{2} \mathrm{SO}_{4}$, and hot $1 \%$ $\mathrm{NaOH}$ fractions had higher carbohydrate contents, they had a significantly lowered prebiotic index because hydrolysis of FOS is known to occur at $\mathrm{pH}<6.0$ and at $\mathrm{pH}>11.0$ [24-26]. Moreover, the extraction temperature increases (up to $35 \%$ ) the solubility of FOS at a temperature of $\sim 90^{\circ} \mathrm{C}[26,27]$, and thus, hot-aqueous fractionations could result in large-scale extraction of FOS than the cold aqueous fractionations. Organic solvents like ethanol, methanol, acetonitrile, and acetone are often used to extract long-chain FOS with acetone or ethanol as precipitating solvents $[28,29]$. Therefore, the higher probiotic index in hot water and hot $80 \%$ ethanol was due to a more significant recovery of FOS. According to Huebner et al. [30], the prebiotic index is directly concerned with the ability of a given substrate to promote the growth of probiotics compared with other non-probiotics and/or pathogenic organisms. Thus, it signifies a particular substrate's ability to be considered prebiotics for selective stimulation of the targeted probiotics organisms. Therefore, two selected carbohydrate fractions (hot-water and $80 \%$ ethanol fractions) having a higher prebiotic index were used for subsequent characterization and the prebiotic parameter studies. 


\subsection{Chemical characterization of the selected carbohydrate fractions}

\subsubsection{FT-IR analyses of the carbohydrate fractions}

The FT-IR spectra of the carbohydrate fractions were shown in Fig. 2. Both the carbohydrate fractions show some similarity in the spectral bands, though the intensity was different. The spectral analysis shows that the strong absorption bands within the range of $3433-3419 \mathrm{~cm}^{-1}$ were due to stretching vibrations of hydroxyl groups [31]. Peaks in the range $3022-3014 \mathrm{~cm}^{-1}$ correspond to the symmetric stretching vibrations of the $=\mathrm{C}-\mathrm{H}$ group. Peaks in the range 2802-2809 $\mathrm{cm}^{-1}$ correspond to the symmetric stretching vibrations of the C-H group. The strong peak at the $1696-$ $1700 \mathrm{~cm}^{-1}$ range indicates the presence of the $\mathrm{C}=\mathrm{O}$ group $[18,32]$; bands at $1452-1315 \mathrm{~cm}^{-1}$ range depict the $-\mathrm{C}-\mathrm{H}$ group. Peaks at $1241-1214 \mathrm{~cm}^{-1}$ span indicated C-N groups, and the strong bands at $1056-1006 \mathrm{~cm}^{-1} \mathrm{correspond} \mathrm{to}^{-10}$ C-O-C unsymmetrical stretching, glycosidic bridge, and presence of the manno-pyranose compound. Peaks in the $950-1200 \mathrm{~cm}^{-1}$ region are the fingerprint region of all polysaccharides [33]. The characteristic peaks at $816-776 \mathrm{~cm}^{-1}$ indicated the existence of $\beta$-glycosidic bonds. The peak at $803 \mathrm{~cm}^{-1}$ was attributed to D-mannose in pyranose, and $735-500 \mathrm{~cm}^{-1}$ shows the presence of any R-CH group [34]. Based on the above data interpretation, it can be concluded that the selected carbohydrate fractions were composed of sugar units in $\beta$-configuration (Fig. S4).

\subsubsection{The HPTLC analyses of the carbohydrate fractions}

The HPTLC analysis of the carbohydrate fractions was shown in Fig. 3. The $R f$ value comparison with the standard monosaccharides such as D-fructose, D-fucose, D-galactose, D-glucose, D-mannose, and D-xylose proves that the carbohydrate fractions contained mono or oligomers. However, the presence of L-arabinose and D-rhamnose was not detected in the samples. The faint bands on the sample lane (L5) below the monosaccharide separation level indicate the presence of low-degree polymerizing (DP) oligosaccharides in the sample carbohydrate fractions. This indicates that the carbohydrate fractions contained some low DP oligosaccharides of D-fucose, D-galactose, D-glucose in hotwater fraction, D-glucose, D-fructose, and D-mannose in $80 \%$ ethanol fraction. Hence, from the FT-IR and HPTLC analyses, it can be interpreted that the carbohydrate fractions contained some $\beta$-linked oligosaccharides that are responsible for the prebiotic efficacy of the fractions.

\subsubsection{Electrospray Ionization- Mass Spectrometry (ESI-MS)}


Molecular mass determination by ESI-MS studies of the hot water and $80 \%$ ethanol fractions in positive ionization mode showed the peak similarities among samples comparable to standard prebiotic inulin (Fig. 4A-C). The peak with molecular mass $(\mathrm{m} / \mathrm{z})$ of $364,526,419,463,507,551,595,639$, and 688 in both the fractions was similar, with inulin indicating the compositional similarity between these carbohydrate fractions containing fructooligosaccharides (FOS). Apart from the above similarity peaks, some other molecular peaks of $\sim 133,149,300,375,379$, and 399 in hot water fraction and $80 \%$ ethanol fraction indicate low DP oligosaccharide fractions other than inulin. A similar observation was noted in Prosek et al. [35].

\subsection{In vitro prebiotic properties of the FOS fractions}

The result showed that the LAB strains could effectively remove cholesterol from the culture broth, as shown in Fig. 1b. It was found that in the absence of prebiotics, the residual cholesterol content in the spent broth for LAB-17 and LAB B1.1 was $55.94 \mu \mathrm{g} / \mathrm{ml}$ and $59.89 \mu \mathrm{g} / \mathrm{ml}$, respectively, while in the presence of FOS fractions (i.e. hot water, and $80 \%$ ethanol), inulin (standard), and in combination of hot water and $80 \%$ ethanol carbohydrate fractions, the estimated residual cholesterol content was much lower. It was observed that the residual cholesterol content in the spent broth was the least in the synbiotic combination with both probiotics strains. Thus, the result signifies that the selected prebiotic fractions could reduce serum cholesterol if used in in vivo models either singly or synbiotic preparations.

The DPPH radical scavenging antioxidant activities of the hot water and $80 \%$ ethanol fractions showed that the $\mathrm{IC}_{50}$ of hot water and $80 \%$ ethanol fractions were $36.51 \mu \mathrm{g} / \mathrm{ml}$ and $33.12 \mu \mathrm{g} / \mathrm{ml}$ compared to standard antioxidant, ascorbic acid $(20.85 \mu \mathrm{g} / \mathrm{ml})$ (Table S1). So, these carbohydrate fractions also have antioxidant efficiency that may benefit the target host against oxidative stress.

\subsection{In vivo health beneficial potentials in Swiss Albino mice model}

All the mice of the experimental sets (control and treated) did not show any sign of allergic lesion, sickness, and unusual stool and urine discharge and appeared healthy and fit throughout the feeding experimentation; hence, the treated samples are not toxigenic to the animal model. Despite any significant differences in total food intake among the mice, the growth (body weight) was better in the treatment sets than in the control. The weight gain was maximum in the synergistic synbiotic setup comprising both the probiotics, i.e., LAB-17 and LAB B1.1, and FOS fractions recognizable body weight increment was also observed in the other treatment sets (Fig. 5A). 
The fermentable fibres in the carbohydrate fractions have been reported to influence the nutrient uptake and mineral absorption among the treated mice, as fermentable inulin-type fructans can increase the mineral absorption and positively influence endocrine function related to appetite and satiety [36]. The blood sugar level in all treatment sets was average or significantly decreased, showing the FOS fractions' hypoglycemic effects. Zaky [37] concludes that inulin and FOS are considered as ameliorating agents against hyperglycemia. The lipid profile analysis of treated mice shows that the concentration of total serum cholesterol, serum LDL, serum VLDL, and triglyceride lowered significantly in prebiotics treated sets individually or in combination, compared to the commercial prebiotic Inulin (Fig. 5A, B). The synbiotic combinations of FOS from hot water and $80 \%$ ethanol fractions with probiotics, i.e., LAB17 \& LAB B1.1, also showed a significantly lower cholesterol profile than untreated control mice. At the same time, serum HDL level increases significantly in all the treatment cases compared to untreated control. These results also prove more pronounced hypocholesterolemic effects in synbiotic sets of the carbohydrate fractions than other sets, comparable to the inulin treated set. Therefore, our study explored that the FOS from the hot water and $80 \%$ ethanol fractions isolated from D. alata L. contain some prebiotics that has a synbiotic effect on the serum lipid profile of mice. Lowering high serum cholesterol levels contributes to the prevention of atherosclerosis. Kim \& Shin [38] reported that administration of inulin, a commercially established prebiotic, for 30 days' treatment increased serum HDL in rats compared to control, which is mainly attributed to the reduced absorption of cholesterol and by the higher rate of catabolism in the liver contributed to the hypocholesterolemic effect. Russo et al. [39] proved the efficacy of inulin against gastrointestinal mobility and lipid and glucose metabolism. The blood sugar level in Fig. 5B implicated no abrupt changes in blood sugar level in all treatment sets compared to the untreated control set when checked randomly. Data represents that hot water fraction and $80 \%$ ethanol fraction do not impart the normalized blood sugar level upon their feeding as in inulin treated sets.

\subsubsection{Mammalian toxicity}

Mammalian toxicity tests performed by measuring the serum SGPT and SGOT level in the blood samples of mice treated with inulin, FOS fractions in different combinations along with probiotics, LAB-17 \& LAB B1.1 and compared with the untreated set showed that a significant decrease of SGPT and SGOT in the serum samples of prebiotic and synbiotic treated mice compared to control (Fig. 5B). Serum SGPT and SGOT levels are considered significant markers of parenchymal liver damage caused by toxic substances [18]. Upon treatment in the different experimental 
sets of mice, the level of these two enzymes remained almost the same or slightly decreased than the untreated control set, and the inulin treated set indicating the nontoxic nature of the prebiotic fractions. Thus, the results also support the nontoxic nature of the prebiotic carbohydrate fractions along with the probiotics strains (LAB-17 \& LAB B1.1).

\subsubsection{Effect on Immunoglobulin A level}

Ig A approximately constitutes $15 \%$ of all immunoglobulins in healthy and is well known for its crucial role in the immune function of the mucous membranes. The Ig A levels in the different treated sets (Fig. 5C) show that amount of Ig A had increased in the set of mice treated with inulin and prebiotics fractions alone. Moreover, synbiotic combinations with probiotics and prebiotic fractions resulted in maximum IgA levels. Hence, the study also shows a significantly improved mucosal immune response of the host. A gastrointestinal environment with healthy microflora plays a critical role in body immunity development. The application of insulin and oligofructose protects against pathogens and toxins by beneficially affecting Gut-associated Lymphoid tissues (GALT) and increasing IgA and IgG levels [40]. Thus, these FOS from D. alata L. tuber could improve the gut-immune health of the consumers.

\subsubsection{Statistical significance}

Therefore, these prebiotic FOS fractions and probiotics LAB strains could be a new combination that can be used for hypocholesterolemic therapy in human consumption. Moreover, the renal toxicity test, like serum creatinine level in the prebiotic and synbiotic treated sets, showed no significant changes compared to the untreated test (Fig. 5B). Hence, a high creatinine level in the blood signifies kidney dysfunction, an indicator of renal toxicity [41]. So, these results also indicated the nontoxic nature of the prebiotics fractions, and probiotics could be trialled for their human consumption and synbiotic therapy following the standard guideline. The principal component analysis (PCA) of the health profiles with synbiotic factors among the intergroup correlations is shown in Fig. 5B. The figure shows that the SGPT, SGOT, and creatinine represent one correlating group, and on the same side, serum cholesterol, LDL, VLDL, and triglycerides representing another correlating group. As per the data, combination expression, no change or slightly decreased level of serum creatinine, SGPT, and SGOT indicating the nontoxic nature of the prebiotics fractions as compared to inulin set and control set and the effect of prebiotics fractions on creatinine, SGPT, and SGOT shows a positive correlation. The prebiotics fractions significantly decreased the serum cholesterol, LDL, VLDL, and triglycerides in all experimental sets than the control in a correlative manner, indicating their hypocholesterolemic activity. Whereas HDL level and average weight gain on the opposite face indicating their 
increased amount in all experimental sets, relates to the health benefiting effect. Blood sugar does not correlate to the other groups that are distantly placed, but no abrupt change in the blood sugar level has been observed in all treated sets, proved the non-digestible nature of the crude prebiotics, and could help in maintaining blood sugar levels.

\subsubsection{In vivo adherence of the probiotic LAB strains to the mice intestine}

To be successful probiotic organisms for human welfare, the target bacterial strains must survive in the gut environment [42]. Therefore, adhesion to the mucosal surfaces of the small intestine, where the flow rates are comparatively high, is considered an essential criterion for colonization of any probiotic strain [2]. SEM analyses of various sets of treated mice intestine explored sufficient bacterial colonization of the targeted probiotic strains in the Swiss albino mice (Fig. 6). Maximum colonization of the LAB strains was observed in the gut of mice treated with both LAB-17 and LAB B1.1 along with the prebiotic FOS from the hot water and 80\% ethanol fractions (Fig. 6C, D), compared to treatment set with only LAB-17 and LAB B1.1 strains along with inulin (Fig. 6B). On the other hand, a meagre amount of bacterial colonization was noticed in the gut of untreated mice (Fig. 6A). Hence, it can be said that these prebiotic carbohydrate fractions helped in more adherence of the probiotic LAB strains to the mice intestine and thus providing an added advantage to the host for extended health benefits. A similar observation was noted by Kolida et al. [33] and Menne and Guggenbuhl [44], where the bifidogenic role of inulin and short-chain oligofructose has been investigated. The combination of inulin, fructooligosaccharides, and galactooligosaccharides is also known to be the best infant formula for bifidogenic effects $[45,46]$.

\section{Conclusion}

FOS is a versatile carbohydrate constituent that has been used in alleviating many diseases, including cancer $[6,9,13$, 47-49]. It has also been used in many pharmaceutical formulations, coating materials, and others [7, 39, 50]. The impacts of prebiotic FOS on the gastrointestinal microbiota are emerging, and their effectiveness relies on the composition and function of gut microbiota to benefit human health. The development of potential prebiotics from inexpensive and abundant food sources like commonly available edible tuber parts of plants might provide a valuable source for economic and global use in human and animal health benefits.

D. alata L., a prevalent plant known for its multifaceted medicinal and pharmaceutical properties, abundantly grow in different continents may provide such invaluable health beneficial prebiotic source. Chemical analysis revealed that 
the carbohydrate fractions from the tuber of D. alata L. are rich in inulin-like FOS with an excellent prebiotic activity score and potent hypocholesterolemic and antioxidant properties. The prebiotic potential of the carbohydrate fractions is comparable to well-established market-borne high-cost prebiotic inulin. In vivo application of these FOS fractions to Swiss albino mice proved their immune-enhancing with good lipid-profile attributing potentialities either singly or in synbiotic combination with those probiotics lactic acid bacteria. Furthermore, significant gut colonization enhancement in the presence of prebiotic fractions proves its extended synbiotic efficacy. Thus, the study concludes that the low-cost edible tuber of $D$. alata L. could be used as prebiotics in daily life to exert a hypocholesterolemic effect in obese persons. Further molecular characterization may lead to reporting the exact molecular structure of the novel prebiotic for commercial exploitation of this food source.

\section{Acknowledgement}

The authors are grateful to CSIR-CMERI, Durgapur, West Bengal, India for ESI-MS, FT-IR, HPTLC, and SEM studies.

\section{Declarations}

Funding: Not applicable

Conflicts of interest/Competing interests: The authors declare that they have no known competing financial interests or personal relationships that could have influenced the work reported in this paper.

Availability of data and material: The data that support the findings of this study are available from the corresponding author upon reasonable request.

Code availability: Not applicable

Authors' contributions: Biplab Bandyopadhyay: Data curation; Formal analysis; Investigation; Methodology; Validation; Writing-original draft. Prashanta Kumar Mitra: Statistical analysis and data analysis for its presentation. Vivekananda Mandal: Conceptualization; Formal analysis; Resources; Software; Supervision; Validation; Visualization; Writing-review \& editing. Narayan C. Mandal: Conceptualization; Funding acquisition; Project administration; Resources; Supervision; Visualization; Writing review \& editing. 
Ethics approval: The animal experimentation has been conducted following the Institutional Animal Ethics Committee guideline, Visva-Bharati University, India (vide Ref. No.: IACE/VB/2018/6; Dated: 24-04-2018).

Consent to participate: Not applicable

Consent for publication: Not applicable

\section{References}

1. Goldsmith JR, Sartor RB (2014) The role of diet on intestinal microbiota metabolism: downstream impacts on host immune function and health, and therapeutic implications. J Gastroenterol, 49,785-98.

2. Slavin J (2013) Fiber and prebiotics: mechanisms and health benefits. Nutrients 5:1417-1435. https://doi.org/10.3390/nu5041417

3. Gibson GR, Hutkins R, Sanders ME, et al (2017) Expert consensus document: The International Scientific Association for Probiotics and Prebiotics (ISAPP) consensus statement on the definition and scope of prebiotics. Nat Rev Gastroenterol Hepatol 14:491-502. https://doi.org/10.1038/nrgastro.2017.75

4. Davani-Davari D, Negahdaripour M, Karimzadeh I, et al (2019) Prebiotics: definition, types, sources, mechanisms, and clinical applications. Foods 8:92. https://doi.org/10.3390/foods8030092

5. Sabater-Molina M, Larqué E, Torrella F, Zamora S (2009) Dietary fructooligosaccharides and potential benefits on health. J Physiol Biochem 65:315-328. https://doi.org/10.1007/BF03180584

6. Wang L, Yang H, Huang H, et al (2019) Inulin-type fructans supplementation improves glycemic control for the prediabetes and type 2 diabetes populations: results from a GRADE-assessed systematic review and doseresponse meta-analysis of 33 randomized controlled trials. $\mathrm{J}$ Transl Med 17:410. https://doi.org/10.1186/s12967-019-02159-0

7. Rößle C, Ktenioudaki A, Gallagher E (2011) Inulin and oligofructose as fat and sugar substitutes in quick breads (scones): a mixture design approach. Eur Food Res Technol 233:167-181. https://doi.org/10.1007/s00217-011$1514-9$ 
8. Le Bourgot C, Apper E, Blat S, Respondek F (2018) Fructo-oligosaccharides and glucose homeostasis: a systematic review and meta-analysis in animal models. Nutr Metab (Lond) 15:9. https://doi.org/10.1186/s12986-018-0245-3

9. Nie Y, Luo F (2021) Dietary fiber: An opportunity for a global control of hyperlipidemia. Oxi Med Cell Long 2021:1-20. https://doi.org/10.1155/2021/5542342

10. Boutron-Ruault M-C, Marteau P, Lavergne-Slove A, et al (2005) Effects of a 3-mo consumption of short-chain fructo-oligosaccharides on parameters of colorectal carcinogenesis in patients with or without small or large colorectal adenomas. Nutri Can 53:160-168. https://doi.org/10.1207/s15327914nc5302_5

11. Bruno-Barcena JM, Azcarate-Peril MA (2015) Galacto-oligosaccharides and colorectal cancer: Feeding our intestinal probiome. J Funct Foods 12:92-108. https://doi.org/10.1016/j.jff.2014.10.029

12. Sonnenburg JL, Bäckhed F (2016) Diet-microbiota interactions as moderators of human metabolism. Nature 535:56-64. https://doi.org/10.1038/nature18846

13. Deng X, Ma J, Song M, et al (2019) Effects of products designed to modulate the gut microbiota on hyperlipidaemia. Eur J Nutr 58:2713-2729. https://doi.org/10.1007/s00394-018-1821-z

14. Moreira Júnior RE, de Carvalho LM, dos Reis DC, et al (2021) Diet-induced obesity leads to alterations in behavior and gut microbiota composition in mice. The $\mathrm{J}$ Nutri Biochem 92:108622. https://doi.org/10.1016/j.jnutbio.2021.108622

15. Chase, M.W., Christenhusz, M.J.M., Fay, M.F., Byng, J.W., Judd, W.S., Soltis, D.E., Mabberley, D.J., Sennikov, A.N., Soltis, P.S. and Stevens, P.F., 2016. An update of the Angiosperm Phylogeny Group classification for the orders and families of flowering plants: APG IV. Bot J the Linn Soc, 181(1), pp.1-20. https://doi.org/10.1111/boj.12385

16. Chandrasekara A, Josheph Kumar $\mathrm{T}$ (2016) Roots and tuber crops as functional foods: a review on phytochemical constituents and their potential health benefits. Int J Food Sci 2016:e3631647. https://doi.org/10.1155/2016/3631647 
17. Zhang Z, Wang X, Liu C, Li J (2016) The degradation, antioxidant and antimutagenic activity of the mucilage polysaccharide from Dioscorea opposita. Carb Poly 150:227-231. https://doi.org/10.1016/j.carbpol.2016.05.034

18. Liu, Y., Li, H., Fan, Y., Man, S., Liu, Z., Gao, W. and Wang, T., 2016. Antioxidant and antitumor activities of the extracts from Chinese yam (Dioscorea opposite Thunb.) flesh and peel and the effective compounds. J Food Sci, 81(6), pp.H1553-H1564. https://doi.org/10.1111/1750-3841.13322

19. Alves RML, Grossmann MVE, Silva RSSF (1999) Gelling properties of extruded yam (Dioscorea alata) starch. Food Chem 67:123-127. https://doi.org/10.1016/S0308-8146(99)00064-3

20. Chou S-T, Chiang B-H, Chung Y-C, et al (2006) Effects of storage temperatures on the antioxidative activity and composition of yam. Food Chem 98:618-623. https://doi.org/10.1016/j.foodchem.2005.06.039

21. Hsu C-K, Yeh J-Y, Wei J-H (2011) Protective effects of the crude extracts from yam (Dioscorea alata) peel on tert-butylhydroperoxide-induced oxidative stress in mouse liver cells. Food Chem 126:429-434. https://doi.org/10.1016/j.foodchem.2010.11.004

22. Liu, X., Lu, K., Yu, J., Copeland, L., Wang, S. and Wang, S., 2019. Effect of purple yam flour substitution for wheat flour on in vitro starch digestibility of wheat bread. Food Chem, 284, pp.118-124. https://doi.org/10.1016/j.foodchem.2019.01.025

23. Bandyopadhyay B, Mandal V, Mandal NC (2021) Partial characterization of novel inulin-like prebiotic fructooligosaccharides of Sechium edule (Jacq.) Sw. (Cucurbitaceae) tuberous roots. J Food Biochem. https://doi.org/10.1111/jfbc.13764

24. Lingyun W, Jianhua W, Xiaodong Z, et al (2007) Studies on the extracting technical conditions of inulin from Jerusalem artichoke tubers. J Food Eng 79:1087-1093. https://doi.org/10.1016/j.jfoodeng.2006.03.028

25. Ronkart SN, Paquot M, Deroanne C, et al (2010) Development of gelling properties of inulin by microfluidization. Food Hydrocol 24:318-324. https://doi.org/10.1016/j.foodhyd.2009.10.009

26. Matusek A, Merész P, Le TKD, Örsi F (2009) Effect of temperature and pH on the degradation of fructooligosaccharides. Eur Food Res Technol 228:355-365. https://doi.org/10.1007/s00217-008-0941-8 
27. Kim Y, Wang SS (2001) Kinetic study of thermally induced inulin gel. J Food Sci 66:991-997. https://doi.org/10.1111/j.1365-2621.2001.tb08224.x

28. $\mathrm{Ku} \mathrm{Y,} \mathrm{Jansen} \mathrm{O,} \mathrm{Oles} \mathrm{CJ,} \mathrm{et} \mathrm{al} \mathrm{(2003)} \mathrm{Precipitation} \mathrm{of} \mathrm{inulins} \mathrm{and} \mathrm{oligoglucoses} \mathrm{by} \mathrm{ethanol} \mathrm{and} \mathrm{other} \mathrm{solvents.}$ Food Chem 81:125-132. https://doi.org/10.1016/S0308-8146(02)00393-X

29. Paseephol T, Small D, Sherkat F (2007) Process optimisation for fractionating Jerusalem artichoke fructans with ethanol using response surface methodology. Food Chem 104:73-80. https://doi.org/10.1016/j.foodchem.2006.10.078

30. Huebner J, Wehling RL, Hutkins RW (2007) Functional activity of commercial prebiotics. Int Dairy J 17:770775. https://doi.org/10.1016/j.idairyj.2006.10.006

31. Purama RK, Goswami P, Khan AT, Goyal A (2009) Structural analysis and properties of dextran produced by Leuconostoc mesenteroides NRRL B-640. Carb Poly 76:30-35. https://doi.org/10.1016/j.carbpol.2008.09.018

32. Cao W, Li X-Q, Liu L, et al (2006) Structure of an anti-tumor polysaccharide from Angelica sinensis (Oliv.) Diels. Carb Poly 66:149-159. https://doi.org/10.1016/j.carbpol.2006.02.034

33. Černá M, Barros AS, Nunes A, et al (2003) Use of FT-IR spectroscopy as a tool for the analysis of polysaccharide food additives. Carb Poly 51:383-389. https://doi.org/10.1016/S0144-8617(02)00259-X

34. Klaus A, Kozarski M, Niksic M, et al (2011) Antioxidative activities and chemical characterization of polysaccharides extracted from the basidiomycete Schizophyllum commune. LWT - Food Sci Technol 44:20052011. https://doi.org/10.1016/j.lwt.2011.05.010

35. Prošek M, Simonovska B, Golc-Wondra A, et al (2003) Use of HPTLC for quantitative evaluation of inulin in food products. JPC-J Planar Chromat 16:58-62. https://doi.org/10.1556/JPC.16.2003.1.12

36. Rafter J, Bennett M, Caderni G, Clune Y, Hughes R, Karlsson PC, Klinder A, O'Riordan M, O'Sullivan GC, Pool-Zobel B, Rechkemmer G (2007) Dietary synbiotics reduce cancer risk factors in polypectomized and colon cancer patients. The Am J Clin Nutri 1;85(2):488-96. https://doi.org/10.1093/ajcn/85.2.488

37. Zaky EA (2009) Physiological response to diets fortified with Jerusalem artichoke tubers (Helianthus tuberosus L.) powder by diabetic rats. Am-Eurasian J Agri Environ Sci 5:682-688 
38. Kim M, Shin HK (1998) The water-soluble extract of chicory influences serum and liver lipid concentrations, cecal short-chain fatty acid concentrations and fecal lipid excretion in rats. The J Nutri 128:1731-1736. https://doi.org/10.1093/jn/128.10.1731

39. Russo F, Riezzo G, Chiloiro M, et al (2010) Metabolic effects of a diet with inulin-enriched pasta in healthy young volunteers. Current Pharma Design 16:825-831. https://doi.org/10.2174/138161210790883570

40. Meyer D, Stasse-Wolthuis M (2009) The bifidogenic effect of inulin and oligofructose and its consequences for gut health. Europ J Clin Nutri 63:1277-1289. https://doi.org/10.1038/ejcn.2009.64

41. Olaniyan, M.F. and Okunola, P.O., 2019. Biochemical alterations in plasma total bile acid, creatinine, sodium, potassium, chloride, and bicarbonate in rabbits overdosed with ibuprofen and supplemented with guava leaf (Psidium guajava) extracts. BLDE University J Health Sciences, 4(1), p.11. DOI: 10.4103/bjhs.bjhs_24_18

42. Ouwehand AC, Kirjavainen PV, Shortt C, Salminen S (1999) Probiotics: mechanisms and established effects. Int Dairy J 9:43-52. https://doi.org/10.1016/S0958-6946(99)00043-6

43. Kolida S, Meyer D, Gibson GR (2007) A double-blind placebo-controlled study to establish the bifidogenic dose of inulin in healthy humans. Europ J Clin Nutri 61:1189-1195. https://doi.org/10.1038/sj.ejcn.1602636

44. Menne E, Guggenbuhl N, Roberfroid M (2000) Fn-type chicory inulin hydrolysate has a prebiotic effect in humans. The J Nutri, 130(5), pp.1197-1199. https://doi.org/10.1093/jn/130.5.1197

45. Knol J, Scholtens P, Kafka C, et al (2005) Colon microflora in infants fed formula with galacto- and fructooligosaccharides: more like breast-fed infants. J Ped Gastro Nutri 40:36-42

46. Sook-He, K., Lee, D. and Meyer, D., 2007. Supplementation of infant formula with native inulin has a prebiotic effect in formula-fed babies. Asia Pacific J Clin Nutri, 16(1), p.172.

47. Mazraeh R, Azizi-Soleiman F, Jazayeri SMHM, Noori SMA (2019) Effect of inulin-type fructans in patients undergoing cancer treatments: A systematic review. Pak J Med Sci 35:. https://doi.org/10.12669/pjms.35.2.701

48. Habib NC, Honoré SM, Genta SB, Sánchez SS (2011) Hypolipidemic effect of Smallanthus sonchifolius (yacon) roots on diabetic rats: Biochemical approach. Chemico-Biological Interact 194:31-39. https://doi.org/10.1016/j.cbi.2011.08.009 
49. Sun S, Araki Y, Hanzawa F, et al (2021) High sucrose diet-induced dysbiosis of gut microbiota promotes fatty liver and hyperlipidemia in rats. The J Nutri Biochem 93:108621. https://doi.org/10.1016/j.jnutbio.2021.108621

50. Hempel S, Jacob A, Rohm H (2006) Influence of inulin modification and flour type on the sensory quality of prebiotic wafer crackers. Eur Food Res Technol 224:335-341. https://doi.org/10.1007/s00217-006-0326-9 


\section{Legends to Figures:}
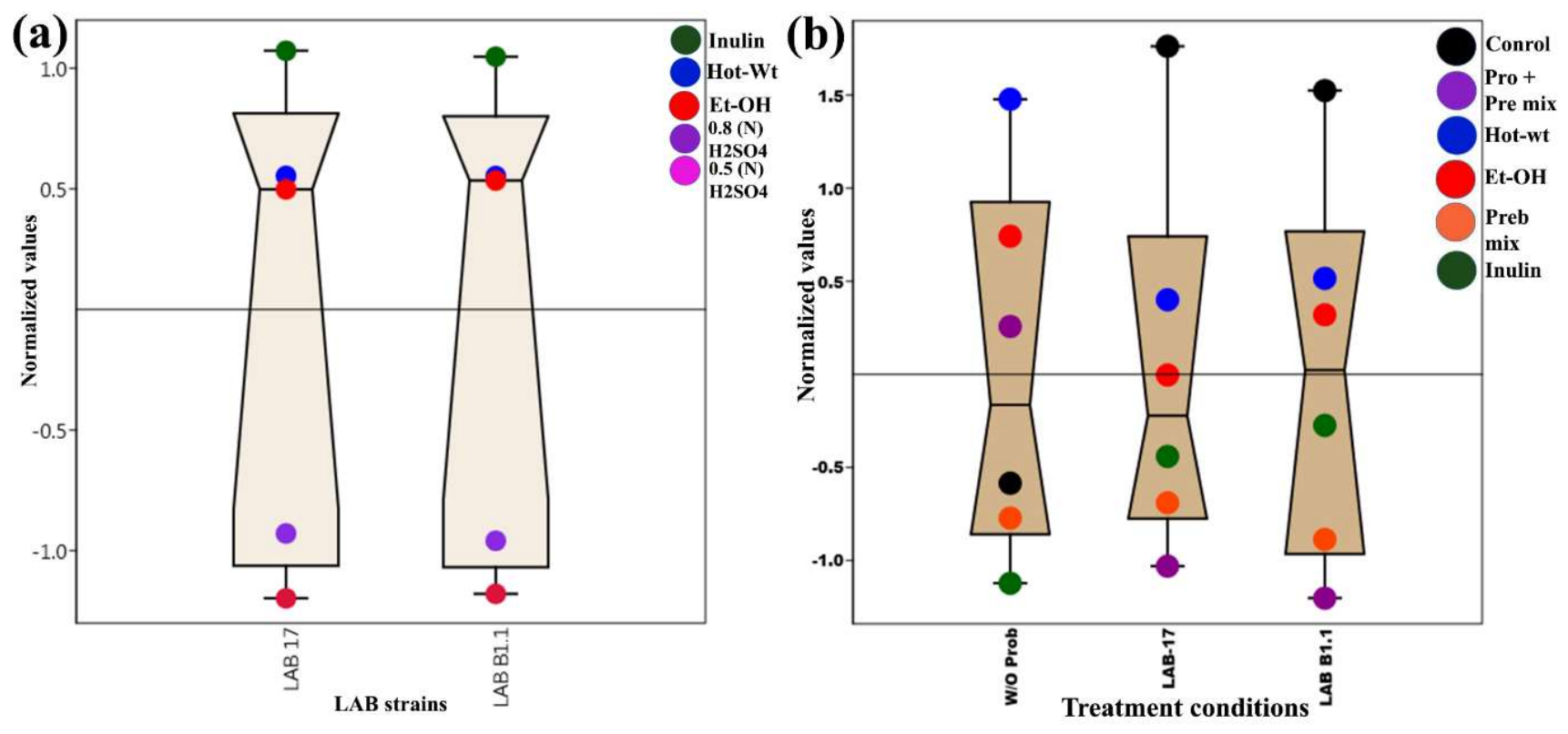

Fig. 1 In vitro selection of the selected carbohydrate fractions. (A) Prebiotic index; and (B) Cholesterol binding. 


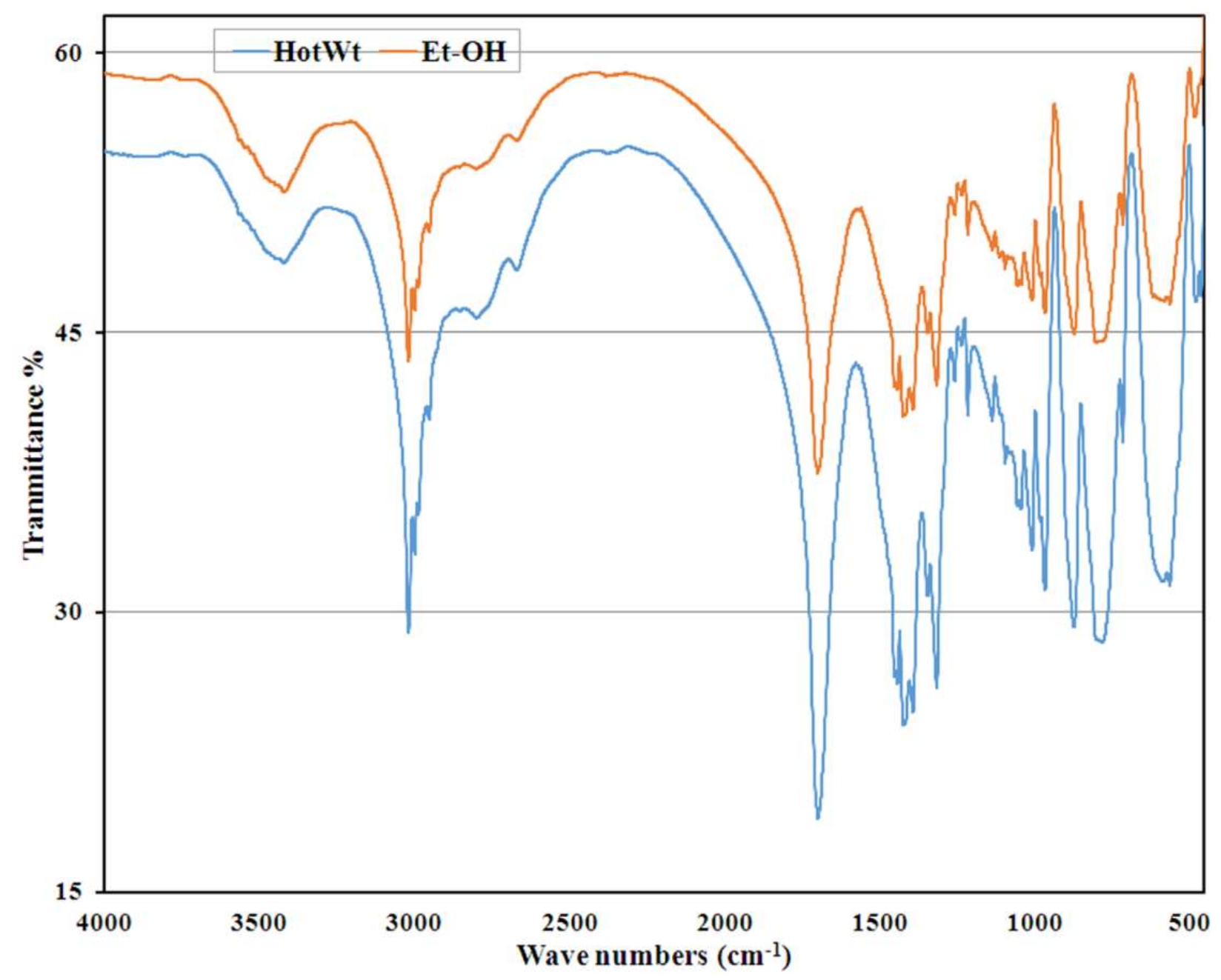

Fig. 2 FT-IR analyses of Hot-Wt and 80\% Et-OH fractions. Here, the blue line indicates the Hot Water fraction, and the red line indicates the $80 \%$ Ethanolic fraction. 

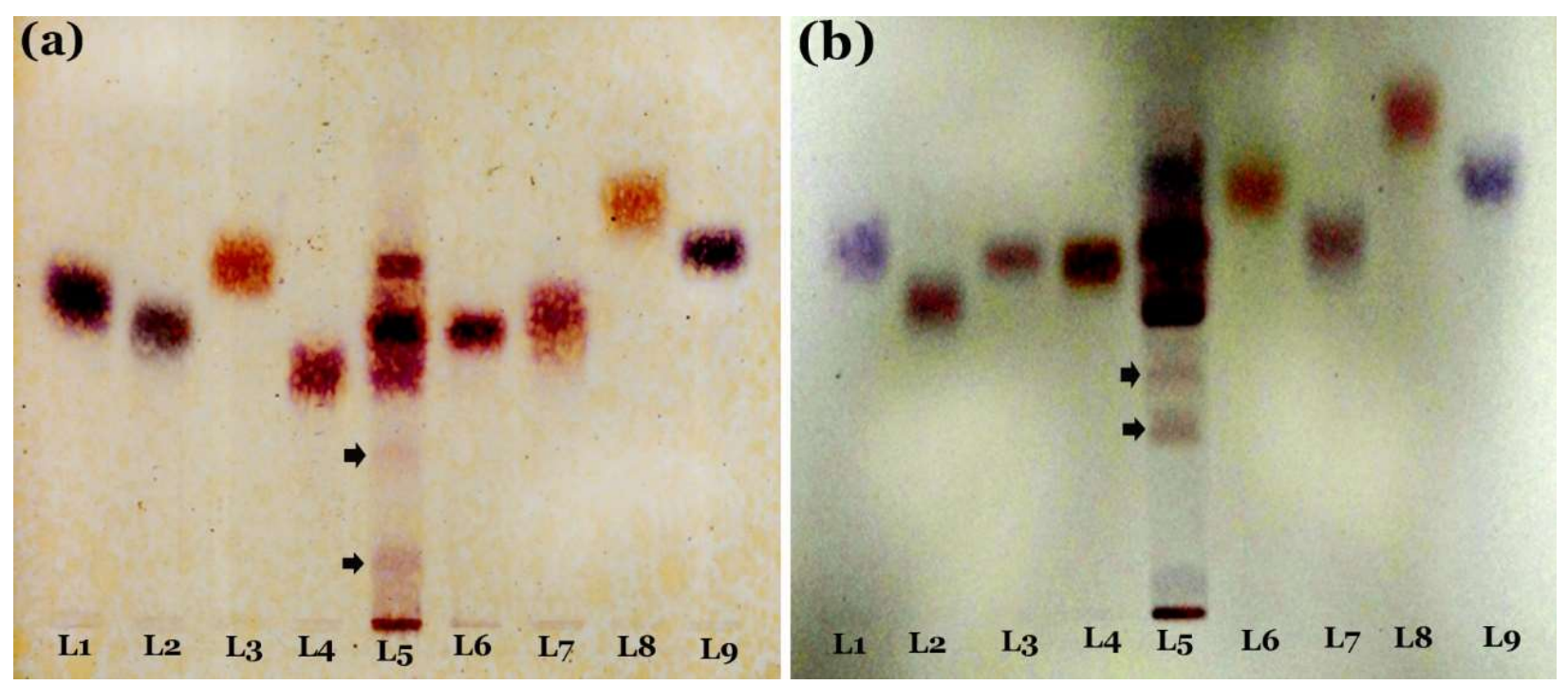

Fig. 3 HPTLC of the fractions. (A) Hot water fraction; and (B) 80\% ethanol fraction. Lanes: L1: L-arabinose, L2: D-fructose, L3: D-fucose, L4: D-galactose, L5: Sample, L6: D-glucose, L7: Dmannose, L8: D-rhamnose, and L9: D-xylose. The arrows in the figure show the oligosaccharides of the fractions. 

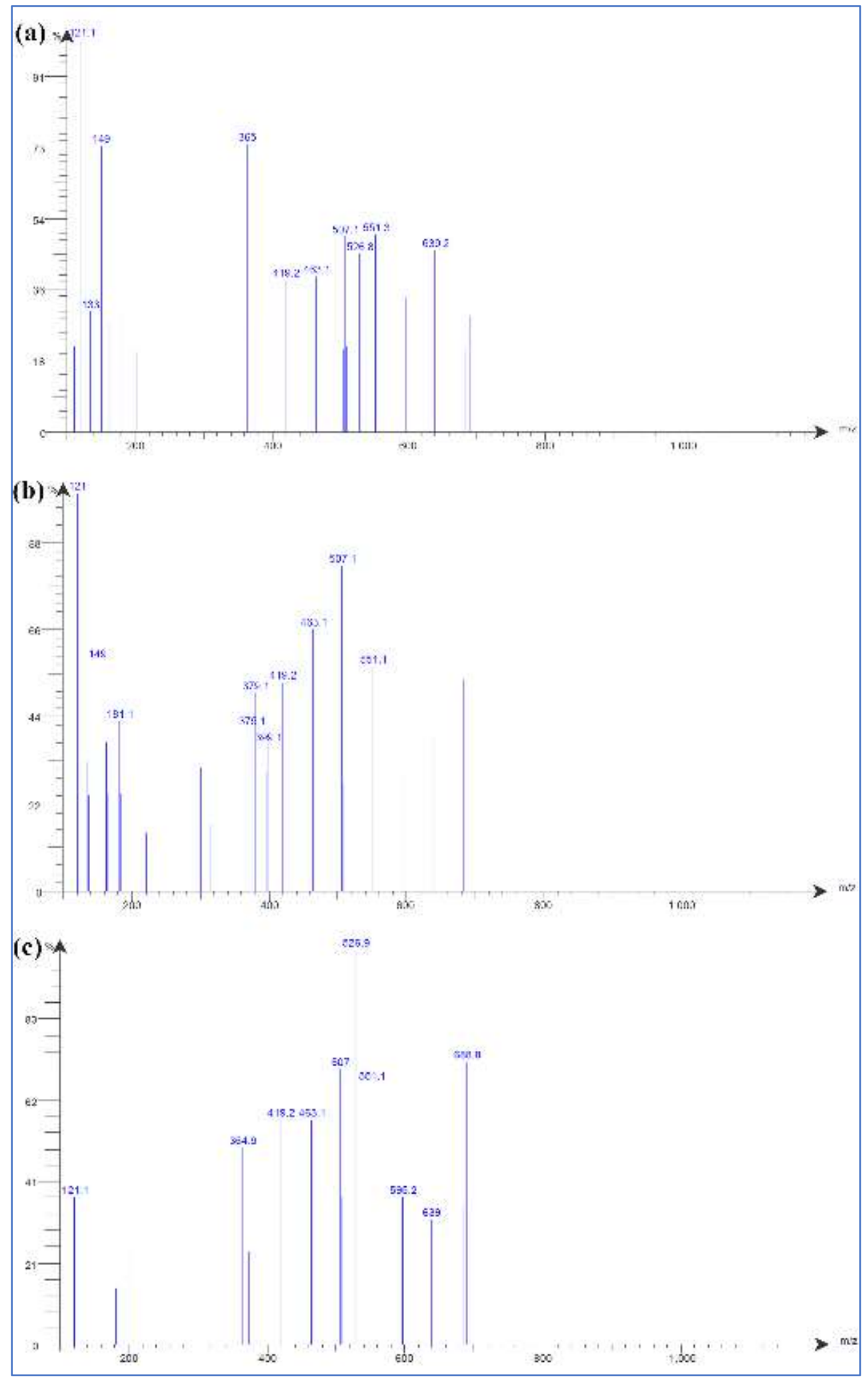

Fig. 4: ESI-MS spectra of the carbohydrate fractions (positive ionization). (A) Hot Water fraction; (B) $80 \%$ Ethanol fraction; and (C) Inulin (HiMedia, RM103). 


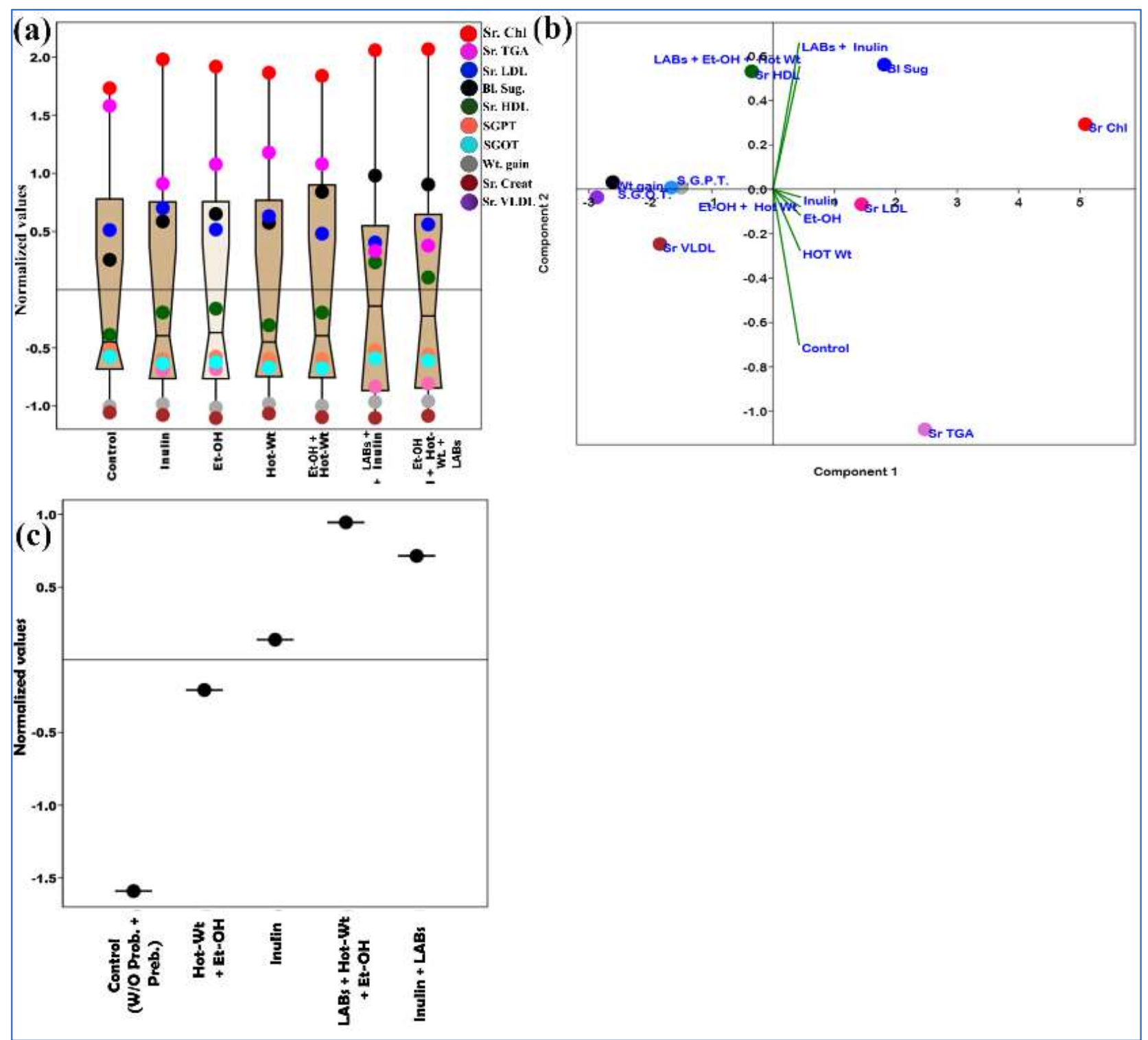

Fig. 5: Health attributes of the different treatment sets. (A) Comparisons of the different health parameters; (B) PCA of the different factors; and (C) Quantitive IgA level in the different treatment sets. 

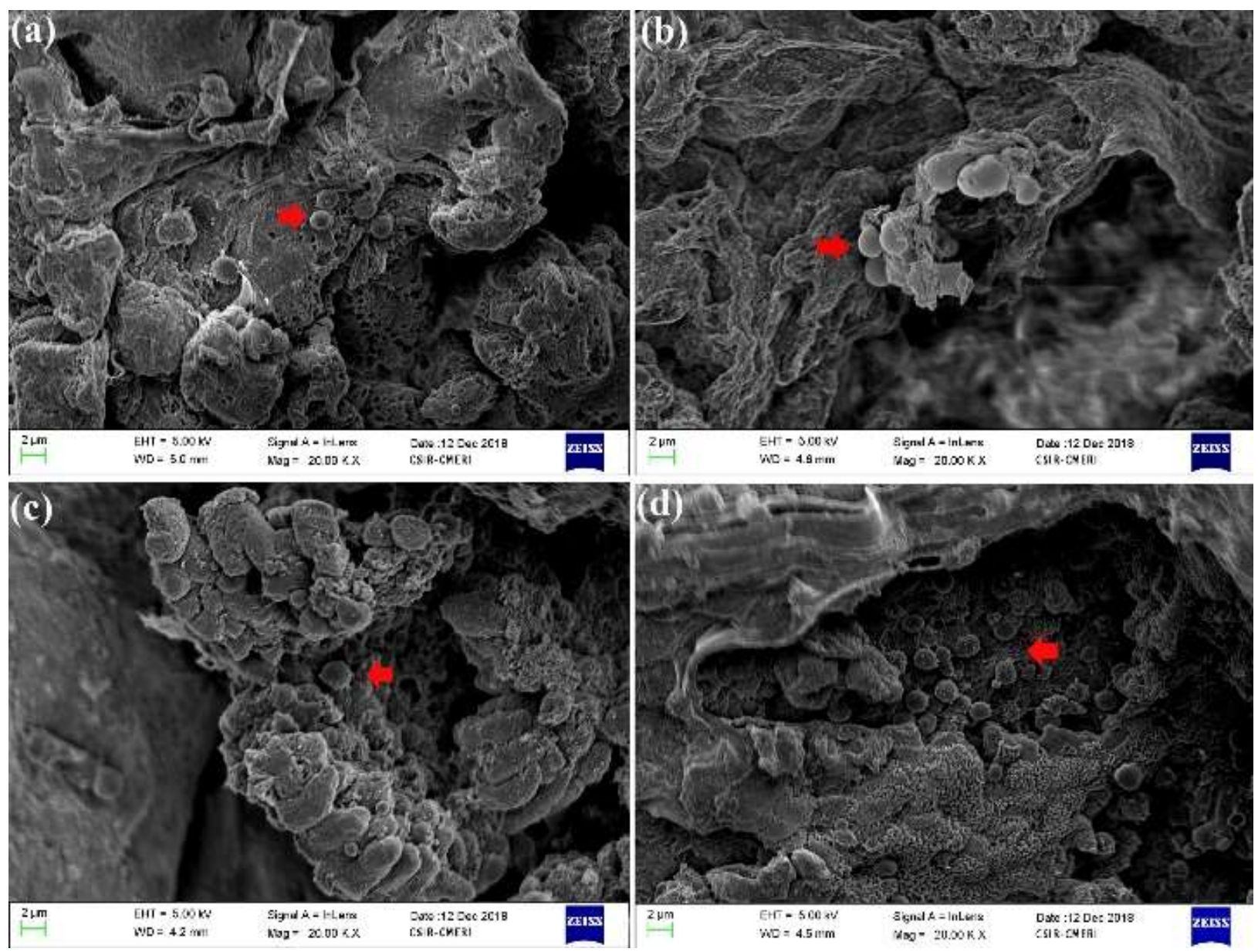

Fig. 6: SEM photomicrographs of the mice intestine treated with different probiotics and synbiotics. (A) Untreated control; (B) treated with Inulin; (C) LAB-17 + LAB B1.1+ Inulin; and (D) treated with LAB-17 + LAB B1.1 + Hot-Wt Fract. + Et-OH fraction. Here, the red arrow indicates bacterial colonization.

\section{Legends to Supplementary Figures:}

Fig. S1: Total extractive carbohydrate content of different treatment fractions of $D$. alata L. (Dioscoreaceae). Here, the error bars are the SE values of the average of triplicate trials.

Fig. S2: In vitro growth enhancement efficacy of the carbohydrate fractions on the probiotic strains for 24 hrs of incubation at $37^{\circ} \mathrm{C}$. Here, the dotted bar indicates the strain LAB-17, and the striped bar indicates the strain LAB B1.1. The error bars are the SE values of the average of triplicate trials. 


\section{Supplementary Files}

This is a list of supplementary files associated with this preprint. Click to download.

- SI2SupplementaryFiguresTables.docx 